
\title{
25 Research Soure \\ Distinct Contributions of the Cerebellum and Basal Ganglia to Arithmetic Procedures
}

\section{William Saban ( $\nabla$ willsaban@berkeley.edu )}

University of California, Berkeley

\section{Pedro Chagas}

Stanford University

Steven Piantadosi

University of California, Berkeley

\section{Rich Ivry}

University of California, Berkeley

\section{Article}

Keywords: Subcortex, Arithmetic, PONT, Neuropsychological studies, Online Platform, Parkinson's disease, Ataxia, Basal Ganglia, Cerebellum

Posted Date: November 30th, 2021

DOI: https://doi.org/10.21203/rs.3.rs-1090281/v1

License: (1) (1) This work is licensed under a Creative Commons Attribution 4.0 International License. Read Full License 


\section{Abstract}

Humans exhibit complex mathematical skills, often attributed to the exceptionally large neocortex. Using a neuropsychological approach, we report that degeneration within two subcortical structures, the basal ganglia and cerebellum, impairs performance in symbolic arithmetic. Moreover, we identify distinct computational impairments in individuals with Parkinson's disease (PD) or cerebellar degeneration (CD). The CD group exhibited a disproportionate cost when arithmetic sum increased, suggesting that the cerebellum is critical for iterative procedures required for calculations. The PD group exhibited a disproportionate cost for equations with an increasing number of addends, suggesting that the basal ganglia are critical for the coordination of multiple cognitive operations. In Experiment 2, the two patient groups exhibited intact practice gains for repeated equations at odds with an alternative hypothesis that these impairments were related to memory retrieval. Overall, the results provide a novel demonstration of the contribution of subcortical structures to the computations required for complex cognition.

\section{Main}

A hallmark of human cognition is our ability to engage in complex reasoning that requires the understanding and utilization of abstract concepts. One powerful example of this is mathematical cognition. While a sense of quantity and simple processes may be observed in many species ${ }^{1-4}$, humans are unique in their ability to engage in symbolic numerical reasoning. Cultural transmission allows high school students to master mathematical concepts that were unknown just a few hundred years ago. Even solving a relatively simple addition problem (e.g., $5+7=12$ ) is a complex cognitive process, requiring several numerical (e.g., counting) and more generic (e.g., memory retrieval) mental processes.

Our understanding of the neural network essential for mathematical cognition has benefitted from the use of the many tools of cognitive neuroscience. One prominent theme is the emphasis on the importance of neocortical regions and in particular, the central role of frontoparietal neocortical regions ${ }^{5-}$ 12. Meta-analyses of functional magnetic resonance imaging (fMRI) data point to the consistent engagement of the inferior parietal lobule and subregions of prefrontal cortex during arithmetic calculations ${ }^{11}$. Developmental imaging studies have shown that the intraparietal sulcus (IPS) is a biomarker of arithmetic skills ${ }^{13,14}$. Correspondingly, neurological patients with damage to these regions exhibit impairments on tests of mathematical cognition ${ }^{15}$. This cortical focus is also manifested in an influential model of mathematical cognition, the Triple-Code model ${ }^{16,17}$. This model highlights the role of three distinct brain regions that perform distinct processes required for numerical abilities: (1) Occipitotemporal areas for processing and encoding Arabic digits; (2) Inferior parietal areas for representing information about quantity and magnitude; 3 ) Peri-sylvian areas that are fundamental to verbal representations of numerical processes (e.g., verbal fact retrieval).

Notably, there has been little discussion of the possible contribution of subcortical regions to mathematical cognition. This is surprising given the expanding appreciation of the contribution of 
subcortical regions to higher-level cognition ${ }^{18-23}$. Indeed, the functional domain of two prominent subcortical structures, the cerebellum and basal ganglia has been recognized to extend well beyond the motor domain ${ }^{24-30}$. Both regions have reciprocal connectivity with many of the cortical areas associated with mathematical cognition $27,31-33$ and, although outside the focus of most neuroimaging studies, activation changes in both the cerebellum and basal ganglia have been consistently observed even in contrasts that control for overt motor responses ${ }^{34-36}$.

In terms of neuropsychological research, research involving individuals with Parkinson's Disease (PD) have generally been descriptive, involving assessments with a range of instruments used to evaluate mathematical abilities ${ }^{37-39}$. In general, basic mathematical processes such as counting, magnitude comparison, and basic arithmetic procedures (e.g., addition, subtraction) appear to be unaffected in PD. The few reports of impairment tend to be on tasks that require solving relatively complex equations, where complexity might be the number of single-digit numbers that can be mentally added or the time required to solve an equation (e.g., $2+5+3+7+6)$. However, these studies have not directly manipulated or even operationalized complexity; as such, they do not provide clear insight into the specific computations impacted by PD.

Even less research has examined how cerebellar dysfunction impacts mathematical cognition. We recently tested individuals with cerebellar degeneration (CD) on a verification task in which the participants had to add or multiply two single-digit numbers. The CD group exhibited an intriguing dissociation: Whereas they exhibited the typical increase in response time (RT) as the calculated sum becomes larger for addition and multiplication problems (i.e., the problem size effect ${ }^{40}$ ), the slope of this function was selectively elevated relative to controls in the addition condition only ${ }^{63}$. We hypothesize that this impairment reflects a slowed rate of movement along a "mental number line", a procedure thought to be essential when adding two, non-identical single digits, but not utilized when performing single-digit multiplication.

In the current study, we take a neuropsychological approach to further explore the role of subcortical regions in mathematical cognition. We compared the performance of individuals with CD, PD and neurotypical control participants on an arithmetic verification task (AVT). We used manipulations in Experiment 1 that allowed us to probe two features of performance on this task that presumably draw on core procedures for addition. First, as noted above, performing addition problems with single digit numbers has been hypothesized to involve a counting procedure that references a mental number line ${ }^{41}$. By this view, the time required to solve the problem will be related to the distance that has to be traversed on this number line. Second, we manipulated the number of required steps, employing problems that involved either two or three operands. Adding up three single-digit numbers will take more time than adding up two single-digit numbers, presumably because the former requires chaining together procedures required for addition.

By testing the $C D$ and PD groups on the same tasks, we can directly evaluate if degeneration of the cerebellum and basal ganglia, respectively, impact performance. Moreover, variation in the pattern of 
impairment between groups will provide insight into how these subcortical structures contribute to mathematical cognition. Although the sparsity of prior work on this problem precludes strong predictions, we expected to replicate our finding of a disproportionately larger problem-size effect in the CD group, suggesting a cerebellar contribution to procedures required for counting. By including two- and threeoperand problems, we can establish if this counting impairment is specific to the magnitude of the step or is also apparent in other procedures such as the number of steps. Given the role of the basal ganglia in a variety of tasks that require chaining together multiple steps ${ }^{42-45}$, we predicted that the PD group would show a disproportionately larger cost on problems involving three addends relative to two addends. It remains to be seen if this group also shows an inflated problem-size effect.

Arithmetic is a practiced skill in most literate adults. Nonetheless, we expect performance will improve over the course of an experiment, reflecting the benefits of short-term practice. An important distinction in the procedural learning literature has been made between benefits that accrue from improved efficiency in algorithmic procedures and benefits that accrue from memory processes ${ }^{46-48}$. In the numerical cognition domain, prior work has shown that short-term benefits can arise from improved efficiency in counting (algorithmic learning) and enhanced memory retrieval ${ }^{49-52}$. Indeed, improvements in these two processes can produce the ubiquitous power function observed in studies of arithmetic, even on a single experimental session ${ }^{47,53,54}$.

We compared practice benefits for the PD, CD, and controls on the AVT in Experiment 2. To measure shortterm practice benefits associated with algorithmic and memory-based learning, we assessed problems that appeared only a single time (No-Repetition condition) and those that were repeated multiple times (Repetition condition). Although the basal ganglia and cerebellum have been associated with procedural learning in a variety of task domains ${ }^{44,55}$, we are unaware of any work looking at the contribution of these subcortical structures to procedural learning in the math domain. An algorithmic-based impairment should be manifest as a selective reduction of practice gains for non-repeated items. In contrast, a memory-based impairment would be evident as reduced practice gains for both non-repeated and repeated items.

\section{Results}

\section{Experiment 1}

Figure 1A shows RT as a function of sum and group, with separate figures provided for the two-addend (simple) and three-addend (complex) problems. We used a linear mixed-effect $\left(\mathrm{LME}^{56}\right)$ model with the factors Group (Control/PD/CD), Complexity (complex/simple), and Sum (3-17), with participant as a random factor. Years of education, age, and MoCA score were included as covariates. We log-transformed the RTs to fit the assumptions of the LME model. Collapsing across conditions, mean RTs were $1482 \mathrm{ms,}$ $1585 \mathrm{~ms}$, and $1829 \mathrm{~ms}$ for the Control, PD, and CD groups, respectively. The CD group was significantly slower than the Control group (est.=.209, $\mathrm{SE}=.055, p<.001$ ) and the PD group (est.=.143, $\mathrm{SE}=.056, p=.014$ ). 
The PD group was slower than the Control group but this difference was not significant (est. $=.066$, $\mathrm{SE}=.054, p=.229)$.

Turning to our mathematical variables of interest, RT increased as a function of both sum and complexity, a pattern that was observed in all three groups. Considering the Control group as establishing baseline performance, this group was, on average, slower to respond to the three-addend problems by $547 \mathrm{~ms}$ compared to the two-addend problems (the "complexity effect"; est.=.381, SE=.005, $p<.001$ ). In terms of the sum effect, this group showed an increase in RT of $43.3 \mathrm{~ms} /$ sum unit (est. $=.0314, \mathrm{SE}=.0008$, $p<.001)$.

Our main focus in Experiment 1 is on the comparison of the effect of complexity and sum between groups (Fig. 1B). The complexity effect (three-addends minus two-addends) was larger for the PD group compared to both the Control group (est. =-.0447, SE=.0074, $p<.001$ ) and CD group (est.=-.0542, SE=.0078, $p<.001)$. The Control and CD groups did not differ on this variable (est. =-.0095, $\mathrm{SE}=.0076, p=.213)$. In contrast, the sum effect was larger for the CD group compared to both the Control group (est. $=.0297$; $\mathrm{SE}=.0038, p<.001)$ and PD group (est. $=.0314, \mathrm{SE}=.0039, p<.001)$. The Control and PD groups did not differ on this variable (est. $=-.0017, \mathrm{SE}=.0037, p=.638$ ). In terms of the covariates, there were no significant effects of education (est. $=.005, \mathrm{SE}=.025, p=.821$ ), age (est. $=.020, \mathrm{SE}=.025, p=.440$ ), or MoCA )est. $=-.007$, $\mathrm{SE}=.026, p=.779)$.

Taken together, two main points can be taken from these results. First, both patient groups exhibited an impairment on the arithmetic verification task, providing novel evidence of the contribution of the cerebellum and basal ganglia to higher cognition. Second, the results point to a double dissociation in terms of how the cerebellum and basal ganglia contribute to algorithmic procedures required for symbolic arithmetic. The CD group showed a selective impairment on the magnitude manipulation, exhibiting a larger slope for the function relating RT to the sum of the digits. In contrast, the PD group showed a bigger cost compared to the other two groups when adding three digits relative to two digits. We hypothesize that the former reflects the involvement of the cerebellum in counting, a procedure that has been hypothesized to entail mental movement along a number line ${ }^{41}$. The added cost incurred by the PD group for complex problems may reflect the involvement of the basal ganglia in chaining together a series of operations ${ }^{42}$ or in facilitating transitions between successive operations (i.e., set switching ${ }^{43}$ ).

\section{Experiment 2}

Figure 2 shows RT as a function of learning cycle and group, with separate functions for the Repetition and No Repetition conditions. In Experiment 2, the LME model included the factors Group (Control/PD/CD), Repetition Condition (Repetition/No repetition), and Cycle (1-12), with participant as a random factor. Years of education, age, and MoCA score were included as covariates.

Collapsing across all conditions, the mean RTs were $1953 \mathrm{~ms}, 2148 \mathrm{~ms}$, and $2281 \mathrm{~ms}$ for the Control, PD, and CD groups, respectively, a pattern similar to that observed in Exp 1. Both patient groups were slower 
than the control group although this effect was only significant for the CD group (CD vs Control: est.=.177, SE=.059, $p=.004$; PD vs Control: est. $=.110, \mathrm{SE}=.062, p=.080)$. The mean RTs for CD and PD groups were not significantly different (est.=.066, $\mathrm{SE}=.063, p=.299$ ).

As expected, the participants got faster at the task over the course of the experiment, and this improvement was especially marked in the Repetition condition. Using the control group to establish baseline performance, this group got faster by $41.4 \mathrm{~ms} /$ cycle for repeated items (est.=-41.4, SE=2.2, $p<.0001$ ) and $26.4 \mathrm{~ms} /$ cycle for non-repeated items (est.=-26.4, SE=2.2, $p<.0001$ ). The difference between these two slopes was significant (est. difference in slope=-14.9, SE=3.1, $p<.0001$ ), consistent with the hypothesis that performance in the Repetition condition benefits from memory retrieval.

Turning to our main analysis (Fig. 2B), there was a significant three-way interaction between Group X Repetition X Cycle (CD vs. Control: est.=-.022, SE=.022, $p=.046$; PD vs. Control: est. $=-.003, \mathrm{SE}=.011$, $p=.766$ ). We compared the learning effect (slope of the function relating RT to Cycle) between the groups separately for the Repetition and No Repetition conditions. For the Repetition condition, the three groups showed a similar change in performance across cycles (CD vs. Control: est. $=.004, S E=.007, p=.531 ; \mathrm{PD}$ vs. Control: est. $=.006, \mathrm{SE}=.007, p=.419$; $\mathrm{CD}$ vs PD: est.=-.001, $\mathrm{SE}=.008, p=.839)$. However, for the No Repetition condition, the $C D$ group showed less improvement over cycles than the Control group (est.=.023, $\mathrm{SE}=.007, p=.002$ ) and the PD group (est.=.016, $\mathrm{SE}=.008, p=.047$ ). The comparison between the Control and PD groups was not significant (est.=.006, SE=.008, $p=.425$ ). Neither education (est. $=.009$, $\mathrm{SE}=.023, p=.670$ ), age (est. $=.005, \mathrm{SE}=.023, p=.815)$, or MoCA score (est. $=-.015, \mathrm{SE}=.024, p=.521$ ) were significant when entered as covariates in the model.

In summary, the results of Experiment 2 show dissociable benefits from different forms of learning. Participants exhibited a large decrease in RT on the equations that repeated multiple times, an effect we assume is driven by the availability of item-specific memory-based retrieval. Given that the magnitude of this effect was similar in all three groups, we assume that the basal ganglia and cerebellum are not essential for the processes required to encode and retrieve the equations. In contrast, the CD group showed a reduced practice benefit in evaluating the novel equations, a signature of what we interpret as an indicative of an impairment in algorithm-based learning. Given the results of Experiment 1, this selective impairment may be related to the counting procedure, here manifest as a problem in becoming more facile in counting over the course of the experimental session.

\section{Discussion}

Converging evidence has highlighted the involvement of the basal ganglia and cerebellum in a broad range of cognitive domains including cognitive control, decision making, and language $23,25,27,34,58-60$. In the present study, we examined the involvement of these subcortical structures in mathematical cognition, a domain in which current models focus on a frontal-parietal cortical network ${ }^{11,61}$. Taking a neuropsychological approach, we tested patients with Parkinson's disease (PD) and cerebellar 
degeneration (CD) on mental addition tasks, using these groups as models to evaluate the role of the basal ganglia and cerebellum, respectively.

The results of Experiment 1 revealed a double dissociation between two arithmetic procedures. Relative to both the Control and PD groups, the CD group showed a larger slope for the function relating RT to the sum of the digits (i.e., problem size effect). In contrast, the PD group showed a larger cost relative to the other two groups when evaluating problems with three addends relative to two addends. These results not only implicate the basal ganglia and cerebellum in mathematical cognition but point to distinct computational contributions. In Experiment 2, we used a learning design that allowed us to examine itemspecific and item-general practice benefits. Both patient groups showed a similar practice benefit as the Control group on repeated equations. Coupled with the double dissociation observed between groups in Experiment 1, this null finding argues against the hypothesis that the patients' impairments might be fully explained by deficit in memory processes. Interestingly, the CD group showed an attenuated practice effect when tested with non-repeated novel equations. This pattern of results underscores a selective contribution of the cerebellum to algorithm-based learning.

Addition problems involving single digits can be solved by memory retrieval or/and by counting procedures ${ }^{40,49,62}$. From a memory perspective, the problem size effect may appear because we have more experience with small size problems (e.g., 3+2) compared to large size problems (e.g., 3+8). From a counting perspective, the problem size effect arises from a process that references a mental number line; thus, adding 8 will take more time than adding 2 because of the additional iterations required by the former. In one variant of this model, these iterations are conceptualized as mental movement along a spatialized number line ${ }^{41}$. Recently, it was found that individuals with CD show a larger problem size effect on addition problems, but not multiplication problems ${ }^{63}$. This previous result points to a role of the cerebellum in counting-like procedure, given that a retrieval problem should impact both types of procedures. The results from Experiment 1 demonstrate the selective cerebellar role in counting (and not in coordination of multiple operations) and points to some degree of specificity in that the PD group did not show a larger problem size effect.

The results from Experiment 2 are also consistent with a counting deficit hypothesis. With repeated items, the demands on counting can be reduced since the answer can be obtained by reference to recently activated memorie ${ }^{47}$. The fact that the CD group showed comparable practice gains for repeated items as the Control and PD groups suggests that this type of memory is intact. However, as suggested by the results of Experiment 1, non-repeated items require more specific mathematical procedures including counting. As such, the CD impairment would continue to be manifest. Moreover, the reduced rate of learning found in Experiment 2 suggests that some of the improvement exhibited by the Control and PD groups for non-repeated items reflects short-term benefits in the rate of counting. We recognize the inferential reasoning underlying this argument; future experiments could directly examine how different arithmetic procedures (i.e., counting, carrying, number of steps) changes over the course of learning. 
To this point, we have focused on the hypothesis that the problem size effect may be reflective of a counting-like procedure. However, one should note that the problem size effect may also arise from a different mathematical procedure, carrying: Problems with larger sums are more likely to require a carry procedure (e.g., $6+7)$ than problems with smaller sums (e.g., $3+4)$. One way to assess the carry procedure effect is to compare equations that either require carrying or not, that are matched in terms of the lower addend (e.g., $5+3$ vs $8+3$ ) given that people tend to increment from the larger digit ${ }^{62}$. Although the sample is limited, for equations matched in this manner, we did observe longer RTs for equations in which the sum was greater than 10. However, we also observed a significant positive slope in RT if we only consider the set of equations that summed to higher than 10 . The latter result underscores that the problem size effect is not solely due to carrying. It would be interesting in future experiments to create balanced stimulus sets to have sufficient power to independently examine the effect of cerebellar damage on carrying and counting.

As noted above, the PD group showed a different pattern of performance than the CD group. Their problem size effect was comparable to that observed for the Control Group. However, relative to both of the other groups, the PD group exhibited a larger cost in adding three single-digit numbers compared to adding two single-digit numbers. While we referred to this manipulation as one of complexity, the twoand three-addend problems involve the same procedures; where they differ is that the latter require additional steps (e.g., add two numbers and that sum becomes an addend to go with the third number). Models of basal ganglia function have highlighted the involvement of this structure in executive functions, including working memory ${ }^{38}$, chaining a series of operations ${ }^{42}$, or facilitating the transition between successive procedures (i.e., set switching ${ }^{43}$ ). The larger complexity effect observed in the PD group would be consistent with these computations: Three-addend problems are likely more taxing on working memory, as well as require performing a longer series of procedures. Future work can use tasks that hone in on different computations; at present, it is intriguing to consider that the impairment observed in the math domain may be indicative of a domain-independent impairment.

Despite the inflated complexity effect observed in the PD group, these individuals showed similar practice benefits as the Controls for both repeated and non-repeated items in Experiment 2. This intact rate of learning suggests that the improvement exhibited by the Control group for non-repeated items is unlikely to be due to short-term benefits in procedures underlying the complexity effect (working memory, chaining, task switching). However, this hypothesis needs to be viewed with caution since it rests on a null result, the absence of a practice deficit in the PD group. Future experiments should directly examine how subcortical (and cortical) contributions to different arithmetic procedures (i.e., counting, carrying, complexity) change over the course of learning.

Models of the brain networks supporting mathematical cognition have focused on the cerebral cortex, and in particular frontoparietal network. The present results point to the need for a broader conceptualization, one that incorporates the basal ganglia and cerebellum. We note that we have only looked on one elementary feature of numerical fluency, the addition of single-digit numbers. A more comprehensive picture will require experiments that examine subcortical contributions to a broad range 
of mathematical procedures (e.g., subtraction, multiplication, geometric relations). The double dissociation between counting and complexity gives reason to expect that this line of research will help reveal how the basal ganglia and cerebellum work in concert with the cortex to support mathematical cognition.

Considered more broadly, the present results provide a novel demonstration of how subcortical systems contribute to higher-level cognition ${ }^{19,21,58,64-66}$. We assume that the computations provided by the cerebellum and basal ganglia for math initially evolved to support more elementary functions, functions that might be performed in the absence of a developed cortex ${ }^{67}$. Coupling these subcortical systems with the expanded representational capacity of the cortex allows for the emergence of complex cognitive representations such as arithmetic. As Paul Rozin has noted, a process that evolved to solve a specific

problem may come to be exploited across a broad range of task domains ${ }^{68}$. In this manner, the functional domain of subcortical regions has expanded, evolving in parallel with the cortex to create novel cognitive competences.

\section{Conclusion}

Converging lines of evidence have challenged a cortico-centric view of complex cognition. Here we add to this literature, exploring a novel cognitive domain, mathematical cognition. Individuals with either degeneration of the basal ganglia or cerebellum exhibited distinct patterns of impairment in an arithmetic verification task. This double dissociation highlights that these subcortical structures make distinct computational contributions to the requisite symbolic procedures for arithmetic. More generally, understanding the constraints on these computations will be necessary for a comprehensive map of cognition, one that embraces the cortex and subcortex.

\section{Methods}

\section{Participants}

An online platform, $\mathrm{PONT}^{69}$, was used to recruit and test the participants. PONT entails five primary steps: 1) Contact support group leaders/web-based platforms to advertise the project; 2) Provide means for interested individuals to initiate contact, a requirement set by our IRB protocol; 3) Conduct interactive, remote neuropsychological assessments; 4) Automated administration of the experimental tasks; 5 ) Provide payment and obtain user feedback.

At the time of this project, there were approximately 183 individuals in the PONT database. For Experiment 1, invitations were sent to 47, 72, and 64 individuals in the Control, CD, and PD groups, respectively. The overall response rate to the first email was approximately $15 \%$, and after a few followups rounds of emails, we reached our goal of at least 20 participants per group. Two participants were excluded based on a failure to respond correctly on the attention probes (1 Control and $1 \mathrm{CD}$ ) and three participants reported connection issues and terminated the program ( 1 Control, $1 \mathrm{CD}$, and $1 \mathrm{PD}$ ). Of those 
who completed the study, we excluded the data from two participants who had accuracy scores at the chance level (1 CD and 1 PD). Thus, the final sample consisted of 20 Control, 17 CD, and 18 PD. The CD group was composed of 10 individuals with a known genetic subtype and 7 individuals with an unknown etiology (idiopathic ataxia). The mean duration since diagnosis for the $\mathrm{CD}$ group was 8.2 years ( $\mathrm{SE}=2.6$ ). The mean duration since diagnosis for the PD group was 6.1 years $(S E=1.0)$. None of the individuals in the PD group had undergone surgical intervention as part of their treatment (e.g., DBS) and all were tested while on their current medication regimen.

The same recruitment procedure was followed in Experiment 2 with a goal of enlisting a minimum of 20 participants per group. One PD participant was excluded based on a failure to respond correctly on the attention, three participants did not complete the study due to connectivity issues ( 1 from each group), and two completed the study but were not included in the analyses due to chance level of performance (1 Control and 1 PD). Thus, the final sample consisted of 20 Control, 20 CD, and 17 PD. The CD group included 12 individuals with a known genetic subtype and 8 individuals with idiopathic ataxia. The mean duration since diagnosis for the $C D$ group was 4.2 years $(S E=1.0)$. The mean duration since diagnosis for the PD group was 8.1 years ( $S E=1.3$ ) and, as in Exp 1, none had undergone surgical intervention as part of their treatment, and all were tested while on their current medication regimen.

All participants provided informed consent under a protocol approved by the institutional review board at the University of California, Berkeley.

\section{Neurological and neuropsychological assessment}

Individuals were invited by email to participate in an online, live interview with an experimenter. After providing informed consent, the participant completed a demographic questionnaire. The experimenter then administered a modified version of the Montreal Cognitive Assessment test $\left(\mathrm{MoCA}^{70}\right)$ as a brief evaluation of cognitive status. For Control participants, the session ended with the completion of the MoCA.

The PD and CD participants continued on to the medical evaluation phase. First, the experimenter obtained the participant's medical history, asking questions about age at diagnosis, medication and other relevant information (e.g., DBS for PD; genetic subtype if known for CD), and a screening for other neurological or psychiatric conditions. Second, the experimenter administered a modified version of the motor section of the Unified Parkinson's Disease Rating Scale, (UPDRS ${ }^{71}$ ) to the PD participants and the Scale for Assessment and Rating of Ataxia (SARA ${ }^{72}$ to the CD participants).

Modifications were made to these assessment instruments to make them better suited for online testing. For the MoCA test, we eliminated the "Alternating Trail Making" item since this requires providing the participant with a paper copy of the task. For the UPDRS and SARA, we modified items that require the presence of a trained individual to ensure safe administration. We eliminated the "Postural Stability task" from the UPDRS since it requires that an experimenter to abruptly pull on the shoulders of the participant. We modified three items on the UPDRS ("Arising from Chair", "Posture", and "Gait"), obtaining self-reports 
from the participant rather than the standard evaluation by the experimenter. Similarly, we obtained selfreports of stance and gait for the SARA rather than observe the participant on these items. For the selfreports, we provided the scale options to the participant (e.g., on the SARA item for gait, $0=$ normal/no difficulty and $8=$ unable to walk even supported). The scores for the MoCA and UPDRS batteries were adjusted to reflect these modifications. For the online MoCA, the observed score was divided by 29 (the maximum online score), and then multiplied by 30 (the maximum score on the standard test). Hence, if a participant obtained a score of 26 , the adjusted score will be $(26 / 29) \star 30$, or 26.9 . The same adjustment procedure was performed for the UPDRS. No adjustment was required for the SARA.

The interview took around 30 minutes for the control participants and 40-60 minutes for the PD and SCA participants. Table 1 provides demographic information for the three groups, as well as the adjusted MoCA, SARA (CD), and UPDRS (PD) scores.

Table 1

Demographic and neuropsychological summary of all groups.

\begin{tabular}{|c|c|c|c|c|c|}
\hline & $\begin{array}{l}\text { Years of } \\
\text { Education }\end{array}$ & $\begin{array}{l}\text { \# of } \\
\text { Females }\end{array}$ & Age & MoCA & Motor assessment \\
\hline \multicolumn{6}{|c|}{ Experiment 1} \\
\hline Control & $\begin{array}{l}17.8 \pm 0.5(14- \\
22)\end{array}$ & 11 & $\begin{array}{l}64.2 \pm 2(40- \\
78)\end{array}$ & $\begin{array}{l}27.9 \pm 0.4(25- \\
30)\end{array}$ & \\
\hline CD & $\begin{array}{l}15.9 \pm 0.9(12- \\
28)\end{array}$ & 14 & $\begin{array}{l}61.7 \pm 2.6(40- \\
81)\end{array}$ & $\begin{array}{l}26.6 \pm 0.4(25- \\
30)\end{array}$ & $10.1 \pm 0.8(2-18.5)$ \\
\hline PD & $\begin{array}{l}16.5 \pm 0.6(12- \\
20)\end{array}$ & 9 & $64 \pm 1(47-75)$ & $\begin{array}{l}26.5 \pm 0.7(25- \\
30)\end{array}$ & $\begin{array}{l}19.4 \pm 1.6(11.3- \\
36.6)\end{array}$ \\
\hline \multicolumn{6}{|c|}{ Experiment 2} \\
\hline Control & $\begin{array}{l}18.3 \pm 0.5(13- \\
22)\end{array}$ & 7 & $\begin{array}{l}66.9 \pm 1.5(51- \\
78)\end{array}$ & $\begin{array}{l}27.7 \pm 0.4(25- \\
30)\end{array}$ & \\
\hline CD & $\begin{array}{l}16.1 \pm 0.9(12- \\
25)\end{array}$ & 9 & $\begin{array}{l}63 \pm 3.2(34- \\
81)\end{array}$ & $\begin{array}{l}26.7 \pm 0.4(25- \\
30)\end{array}$ & $10.4 \pm 1.1(2-19.5)$ \\
\hline PD & $\begin{array}{l}18.3 \pm 0.8(12- \\
25)\end{array}$ & 9 & $\begin{array}{l}67.6 \pm 1.9(49- \\
80)\end{array}$ & $\begin{array}{l}27.4 \pm 0.7(25- \\
30)\end{array}$ & $\begin{array}{l}17.1 \pm 1(10.7- \\
26.9)\end{array}$ \\
\hline
\end{tabular}

\section{Procedure}

The experiments were programmed in Gorilla Experiment Builder ${ }^{73}$ and designed to be compatible with any personal computer. Stimuli were presented as black characters on a white screen. The actual size in 
terms of visual angle varied given that participants used their own computer system, but we chose a font (7 HTML) that is clearly legible on all screens (as determined by testing when developing the system).

Participants were invited by email to participate in an experiment. The email provided an overview of the experimental task and included a link that could be clicked to initiate the experimental session. The instructions emphasized that the participant could start the experiment whenever he or she was ready but should only do so when they could complete the 30-45 minutes session. The link was associated with a unique participant ID, providing a means to ensure that the data were stored in an anonymized and confidential manner. Once activated, the link connected to the Gorilla platform used to run the experimental session. The instructions were provided on the monitor in an automated manner, with the program advancing under the participant's control.

Each experiment involved an arithmetic verification task (AVT). The participant was asked to determine whether the mathematical equation (e.g., $3+2+6=11$ ) presented on the center of the screen was true (press the ' $M$ ' key) or false (press the ' $Z$ ' key). At the start of each trial in the AVT, a black fixation cross appeared in the middle of a white background. After $1000 \mathrm{~ms}$, the fixation cross was replaced by a stimulus display that consisted of the equation. The equation remained on the screen until a response was recorded or until $5000 \mathrm{~ms}$, whichever came first. The instructions emphasized that the response should be performed as quickly and accurately as possible. Visual feedback was presented for $500 \mathrm{~ms}$ above equation, with a green checkmark $(\checkmark)$ or red $X$ indicating if the response was incorrect or correct. If a keypress was not detected after $3500 \mathrm{~ms}$, the participant was presented with the phrase "Respond faster" as feedback.

In Experiment 1, we created ten different sets of 288 equations. Each individual was randomly presented with one of the sets. To minimize the effect of memory, each equation only appeared twice $50 \%$ of which were true). For any given equation, the same digit did not appear twice. In Experiment 1, we examined two procedures involved in solving arithmetic problems: Complexity and counting. As a manipulation of complexity, the equations could either require adding two addends (Simple) or three addends (Complex). The sum of the equations varied from 3 to 17 (e.g., 2+1=3 to 9+8=17), and served as our proxy for variation in counting. For each level of complexity, 144 unique equations were presented. Each individual had three breaks of one minute each. The equations were presented in a random order. The experiment was completed within approximately 25 minutes.

In Experiment 2, we created ten different sets of 296 equations. Each individual was randomly presented with one of the sets. The stimulus set was limited to equations with three addends. For any given equation, the same digit did not appear twice. Eight of these equations constituted the Repeated stimuli; the others constituted the No Repetition stimuli. The experiment consisted of a total of 36 blocks of 16 trials each (576 total trials; $50 \%$ of which were true). Each block was composed of one trial with each of the eight Repeat equations and eight trials with No Repetition (unique) equations. The two types of equations were included to provide measures of two forms of learning, memory-based learning (Repetition equations) and algorithm-based learning (No Repetition equations). We averaged across every 
three blocks to collapse the 36 blocks into 12 cycles. The experiment was completed within approximately 45 minutes.

To ensure that participants remained attentive, we included five "attention probes" that appeared in the start of the experiment or during the experimental block. For example, an attention probe might instruct the participant to press a specific key rather than selecting the "next" button on the screen to advance the experiment (e.g., "Do not press the 'next' button. Press the letter ' $A$ ' to continue"). If the participant failed to respond as instructed on these probes, the experiment continued, but the participant's results were not included in the analysis. After completing the experimental task, each participant answered a feedback questionnaire about their experience of the study (e.g., "How well was the study instructions explained." or "Did all the images display correctly?").

\section{References}

1. Agrillo, C., Piffer, L. \& Bisazza, A. Large Number Discrimination by Mosquitofish. PLoS One 5, e15232 (2010).

2. Agrillo, C., Dadda, M. \& Serena, G. Choice of Female Groups by Male Mosquitofish (Gambusia holbrooki). Ethology 114, 479-488 (2008).

3. Dadda, M., Piffer, L., Agrillo, C. \& Bisazza, A. Spontaneous number representation in mosquitofish. Cognition 112, 343-348 (2009).

4. Leibovich-Raveh, T., Raveh, A., Vilker, D. \& Gabay, S. Magnitude integration in the Archerfish. Sci. Rep. 11, 1-11 (2021).

5. Cohen-Kadosh, R. \& Dowker, A. The Oxford handbook of numerical cognition. in The Oxford handbook of numerical cognition (Oxford University Press., 2015).

6. Zamarian, L., Ischebeck, A. \& Delazer, M. Neuroscience of learning arithmetic-Evidence from brain imaging studies. Neurosci. Biobehav. Rev. 33, 909-925 (2009).

7. Andres, M., Pelgrims, B., Michaux, N., Olivier, E. \& Pesenti, M. Role of distinct parietal areas in arithmetic: An fMRI-guided TMS study. Neuroimage 54, 3048-3056 (2011).

8. Ansari, D. \& Dhital, B. Age-related Changes in the Activation of the Intraparietal Sulcus during Nonsymbolic Magnitude Processing: An Event-related Functional Magnetic Resonance Imaging Study. J. Cogn. Neurosci. 18, 1820-1828 (2006).

9. Grabner, R. H. et al. To retrieve or to calculate? Left angular gyrus mediates the retrieval of arithmetic facts during problem solving. Neuropsychologia 47, 604-608 (2009).

10. Dehaene, S., Molko, N., Cohen, L. \& Wilson, A. J. Arithmetic and the brain. Curr. Opin. Neurobiol. 14, 218-224 (2004).

11. Arsalidou, M. \& Taylor, M. J. Is $2+2=4$ ? Meta-analyses of brain areas needed for numbers and calculations. Neuroimage 54, 2382-2393 (2011).

12. O, G., P, I., H, S. \& A, K. Dissociating neural correlates of cognitive components in mental calculation. Cereb. Cortex 11, 350-359 (2001). 
13. Isaacs, E. B., Edmonds, C. J., Lucas, A. \& Gadian, D. G. Calculation difficulties in children of very low birthweightA neural correlate. Brain 124, 1701-1707 (2001).

14. Emerson, R. W. \& Cantlon, J. F. Continuity and change in children's longitudinal neural responses to numbers. Dev. Sci. 18, 314-26 (2015).

15. Van Harskamp, N. J. \& Cipolotti, L. Selective impairments for addition, subtraction and multiplication. Implications for the organisation of arithmetical facts. Cortex 37, 363-388 (2001).

16. Dehaene, S. \& Cohen, L. Towards an anatomical and functional model of number processing. Mathematical cognition (1995).

17. Dehaene, S., Piazza, M., Pinel, P. \& Cohen, L. THREE PARIETAL CIRCUITS FOR NUMBER PROCESSING. Cogn. Neuropsychol. 20, 487-506 (2003).

18. Saban, W., Sekely, L., Klein, R. M. \& Gabay, S. Monocular channels have a functional role in endogenous orienting. Neuropsychologia (2018). doi:10.1016/j.neuropsychologia.2018.01.002

19. Saban, W., Raz, G., Grabner, R. H., Gabay, S. \& Kadosh, R. C. Primitive visual channels have a causal role in cognitive transfer. Sci. Rep. 11, 8759 (2021).

20. Saban, W., Weinbach, N. \& Gabay, S. Monocular channels have a functional role in phasic alertness and temporal expectancy. Attention, Perception, Psychophys. (2019). doi:10.3758/s13414-01801653-9

21. Saban, W., Sekely, L., Klein, R. M. \& Gabay, S. Endogenous orienting in the archer fish. Proc. Natl. Acad. Sci. 114, 7577-7581 (2017).

22. Saban, W., Gabay, S. \& Kalanthro, E. Acta Psychologica More than just channeling: The role of subcortical mechanisms in executive functions - Evidence from the Stroop task. 1-7 (2017). doi:10.1016/j.actpsy.2017.03.001

23. Buckner, R. L. The Cerebellum and Cognitive Function: 25 Years of Insight from Anatomy and Neuroimaging. Neuron 80, 807-815 (2013).

24. Sokolov, A. A., Miall, R. C. \& Ivry, R. B. The Cerebellum: Adaptive Prediction for Movement and Cognition. Trends Cogn. Sci. 21, 313-332 (2017).

25. Schmahmann, J. D. The cerebellum and cognition. Neurosci. Lett. 688, 62-75 (2019).

26. Balsters, J. H., Whelan, C. D., Robertson, I. H. \& Ramnani, N. Cerebellum and Cognition: Evidence for the Encoding of Higher Order Rules. Cereb. Cortex 23, 1433-1443 (2013).

27. Bostan, A. C. \& Strick, P. L. The basal ganglia and the cerebellum: Nodes in an integrated network. Nat. Rev. Neurosci. 19, 338-350 (2018).

28. Middleton, F. A. \& Strick, P. L. Anatomical evidence for cerebellar and basal ganglia involvement in higher cognitive function. Science 266, 458-61 (1994).

29. Ashby, F. G., Turner, B. O. \& Horvitz, J. C. Cortical and basal ganglia contributions to habit learning and automaticity. Trends Cogn. Sci. 14, 208-15 (2010).

30. OWEN, A. M. et al. FRONTO-STRIATAL COGNITIVE DEFICITS AT DIFFERENT STAGES OF PARKINSON'S DISEASE. Brain 115, 1727-1751 (1992). 
31. I, T., J, R., KE, S. \& RE, P. Changes of cortico-striatal effective connectivity during visuomotor learning. Cereb. Cortex 12, 1040-1047 (2002).

32. Ide, J. S. \& Li, C. shan R. A cerebellar thalamic cortical circuit for error-related cognitive control. Neuroimage (2011). doi:10.1016/j.neuroimage.2010.07.042

33. Milardi, D. et al. The Cortico-Basal Ganglia-Cerebellar Network: Past, Present and Future Perspectives. Front. Syst. Neurosci. 0, 61 (2019).

34. King, M., Hernandez-Castillo, C. R., Poldrack, R. A., Ivry, R. B. \& Diedrichsen, J. Functional boundaries in the human cerebellum revealed by a multi-domain task battery. Nat. Neurosci. 22, 1371-1378 (2019).

35. Ischebeck, A., Zamarian, L., Egger, K., Schocke, M. \& Delazer, M. Imaging early practice effects in arithmetic. Neuroimage 36, 993-1003 (2007).

36. Zago, L. et al. How verbal and spatial manipulation networks contribute to calculation: An fMRI study. Neuropsychologia 46, 2403-2414 (2008).

37. Tamura, I., Kikuchi, S., Otsuki, M., Kitagawa, M. \& Tashiro, K. Deficits of working memory during mental calculation in patients with Parkinson's disease. J. Neurol. Sci. 209, 19-23 (2003).

38. Zamarian, L. et al. Parkinson's disease and arithmetics: The role of executive functions. J. Neurol. Sci. 248, 124-130 (2006).

39. Delazer, M. et al. Number processing and basal ganglia dysfunction: a single case study. 42, 10501062 (2004).

40. Ashcraft, M. H. \& Guillaume, M. M. Chapter 4 Mathematical Cognition and the Problem Size Effect. Psychol. Learn. Motiv. - Adv. Res. Theory 51, 121-151 (2009).

41. Dehaene, S. The neural basis of the Weber-Fechner law: a logarithmic mental number line. Trends Cogn. Sci. 7, 145-147 (2003).

42. D, S., CE, M., S, G., J, S. \& MA, G. The role of dopamine in cognitive sequence learning: evidence from Parkinson's disease. Behav. Brain Res. 156, 191-199 (2005).

43. Meiran, N., Friedman, G. \& Yehene, E. Parkinson's disease is associated with goal setting deficits during task switching. Brain Cogn. 54, 260-262 (2004).

44. Pascual-Leone, A. et al. Procedural learning in Parkinson's disease and cerebellar degeneration. Ann. Neurol. 34, 594-602 (1993).

45. Muslimović, D., Post, B., Speelman, J. D. \& Schmand, B. Motor procedural learning in Parkinson's disease. Brain 130, 2887-2897 (2007).

46. Tenison, C., Fincham, J. M. \& Anderson, J. R. Phases of learning: How skill acquisition impacts cognitive processing. Cogn. Psychol. 87, 1-28 (2016).

47. Logan, G. D. Toward an Instance Theory of Automatization. 95, 492-527 (1988).

48. Rickard, T. C. Bending the Power Law: A CMPL Theory of Strategy Shifts and the Automatization of Cognitive Skills. J. Exp. Psychol. Gen. 126, 288-311 (1997). 
49. Ashcraft, M. H. The development of mental arithmetic: A chronometric approach. Dev. Rev. 2, 213236 (1982).

50. LeFevre, J. A., Sadesky, G. S. \& Bisanz, J. Selection of procedures in mental addition: Reassessing the problem size effect in adults. J. Exp. Psychol. Learn. Mem. Cogn. 22, 216-230 (1996).

51. Campbell, J. I. D. \& Xue, Q. Cognitive arithmetic across cultures. J. Exp. Psychol. Gen. 130, 299-315 (2001).

52. Baroody, A. J. An evaluation of evidence supporting fact-retrieval models. Learn. Individ. Differ. 6, 136 (1994).

53. Logan, G. D. \& Klapp, S. T. Automatizing Alphabet Arithmetic: I. Is Extended Practice Necessary to Produce Automaticity? J. Exp. Psychol. Learn. Mem. Cogn. 17, 179-195 (1991).

54. Tenison, C. \& Anderson, J. R. Modeling the distinct phases of skill acquisition. J. Exp. Psychol. Learn. Mem. Cogn. 42, 749-767 (2016).

55. Doyon, J. et al. Experience-dependent changes in cerebellar contributions to motor sequence learning. Proc. Natl. Acad. Sci. U. S. A. 99, 1017-1022 (2002).

56. Bates, D., Mächler, M., Bolker, B. \& Walker, S. Fitting Linear Mixed-Effects Models Using Ime4. J. Stat. Softw. 67, 1-48 (2015).

57. Bates, D., Mächler, M., Bolker, B. M. \& Walker, S. C. Fitting linear mixed-effects models using Ime4. J. Stat. Softw. 67, (2015).

58. Anatomical Evidence for Cerebellar and Basal Ganglia Involvement in Higher Cognitive Function Author (s): Frank A. Middleton and Peter L. Strick Published by : American Association for the Advancement of Science Stable URL : http://www.jstor.org/stabl. 266, 458-461 (2016).

59. Ashby, F. G., Turner, B. O. \& Horvitz, J. C. Cortical and basal ganglia contributions to habit learning and automaticity. Trends Cogn. Sci. 14, 208-215 (2010).

60. Middleton, F. A. \& Strick, P. L. Basal ganglia and cerebellar loops: motor and cognitive circuits. Brain Res. Rev. 31, 236-250 (2000).

61. Arsalidou, M., Pawliw-Levac, M., Sadeghi, M. \& Pascual-Leone, J. Brain areas associated with numbers and calculations in children: Meta-analyses of fMRI studies. Dev. Cogn. Neurosci. 30, 239250 (2018).

62. Barrouillet, P. \& Thevenot, C. On the problem-size effect in small additions: Can we really discard any counting-based account? Cognition 128, 35-44 (2013).

63. McDougle, S. D. et al. Continuous manipulation of mental representations is compromised in cerebellar degeneration. bioRxiv 2020.04.08.032409 (2021). doi:10.1101/2020.04.08.032409

64. Saban, W., Klein, R. M. \& Gabay, S. Probabilistic versus "Pure" Volitional Orienting: a Monocular Difference. Attention, Perception, Psychophys. 80, (2018).

65. Saban, W., Weinbach, N. \& Gabay, S. Monocular channels have a functional role in phasic alertness and temporal expectancy. Attention, Perception, Psychophys. 81, 752-763 (2019). 
66. Saban, W., Gabay, S. \& Kalanthroff, E. More than just channeling: The role of subcortical mechanisms in executive functions - Evidence from the Stroop task. Acta Psychol. (Amst). 189, 3642 (2018).

67. Güntürkün, O. \& Bugnyar, T. Cognition without Cortex. Trends Cogn. Sci. Xx, 1-13 (2016).

68. Rozin, P. The Selection of Foods by Rats, Humans, and Other Animals. Adv. Study Behav. (1976). doi:10.1016/S0065-3454(08)60081-9

69. Saban, W. \& Ivry, R. B. PONT: A Protocol for Online Neuropsychological Testing. J. Cogn. Neurosci. 1-13 (2021). doi:10.1162/JOCN_A_01767

70. Nasreddine, Z. S. et al. The Montreal Cognitive Assessment, MoCA: A brief screening tool for mild cognitive impairment. J. Am. Geriatr. Soc. 53, 695-699 (2005).

71. Fahn S \& Elton, R. The unified Parkinson's disease rating scale. Recent Dev. Park. Dis. 153-163 (1987).

72. Schmitz-Hübsch, T. et al. Scale for the assessment and rating of ataxia: Development of a new clinical scale. Neurology 66, 1717-1720 (2006).

73. Anwyl-Irvine, A. L., Massonnié, J., Flitton, A., Kirkham, N. \& Evershed, J. K. Gorilla in our midst: An online behavioral experiment builder. Behav. Res. Methods 52, 388-407 (2020).

\section{Figures}


(A)
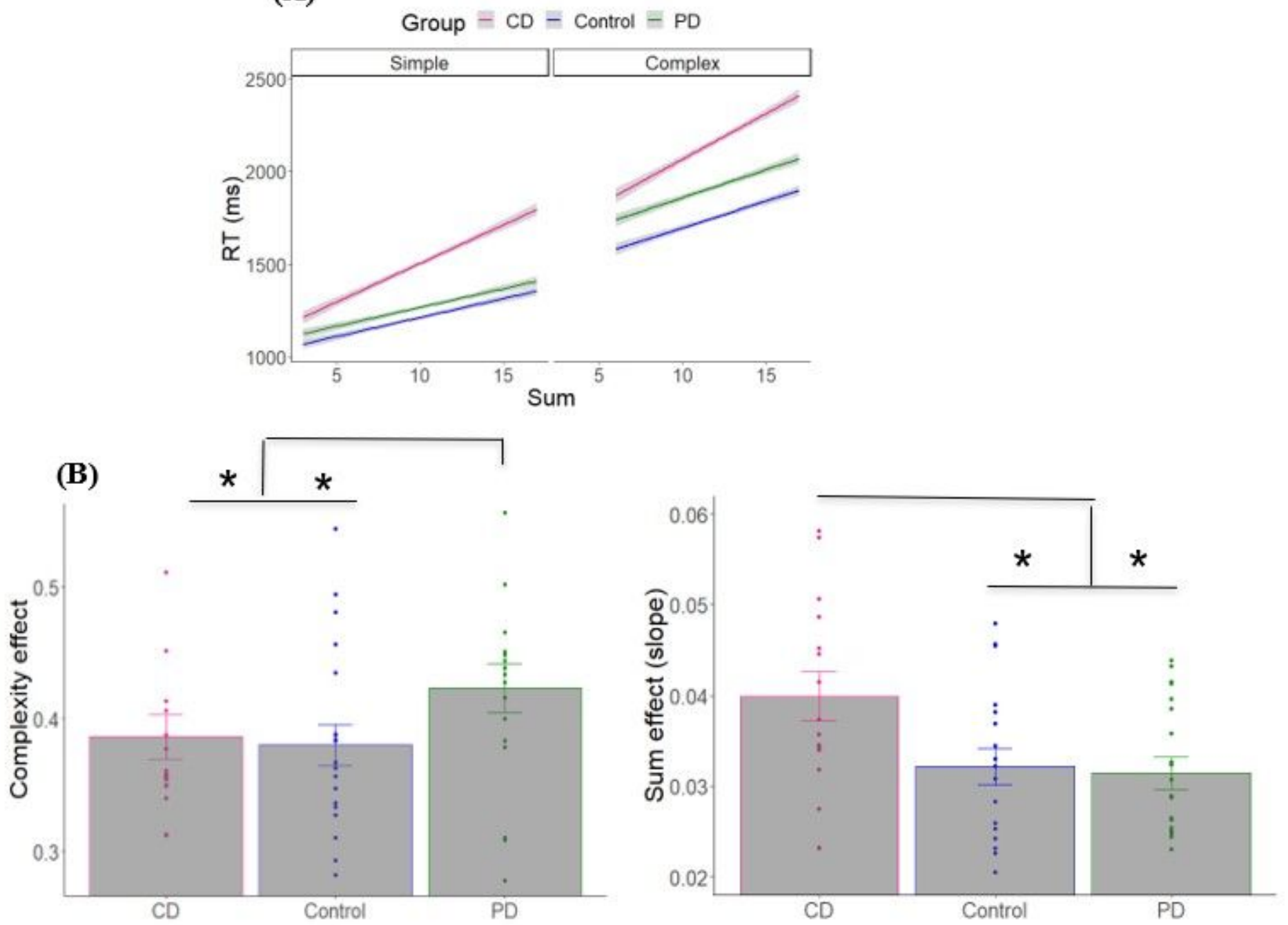

Figure 1

(A) RT as a function of sum for two-addend problems (left) and three-addend problems (right). Error bars $=95 \%$ confidence interval. (B) Left panel shows the effect of complexity, defined as the difference in RT for the three- and two-addend problems. Right panel shows the effect of sum, defined as the slope of the function relating RT to the sum. Dots indicate performance of each individual participant. Error bars $=$ SEM; ${ }^{*}=p<.001$. 

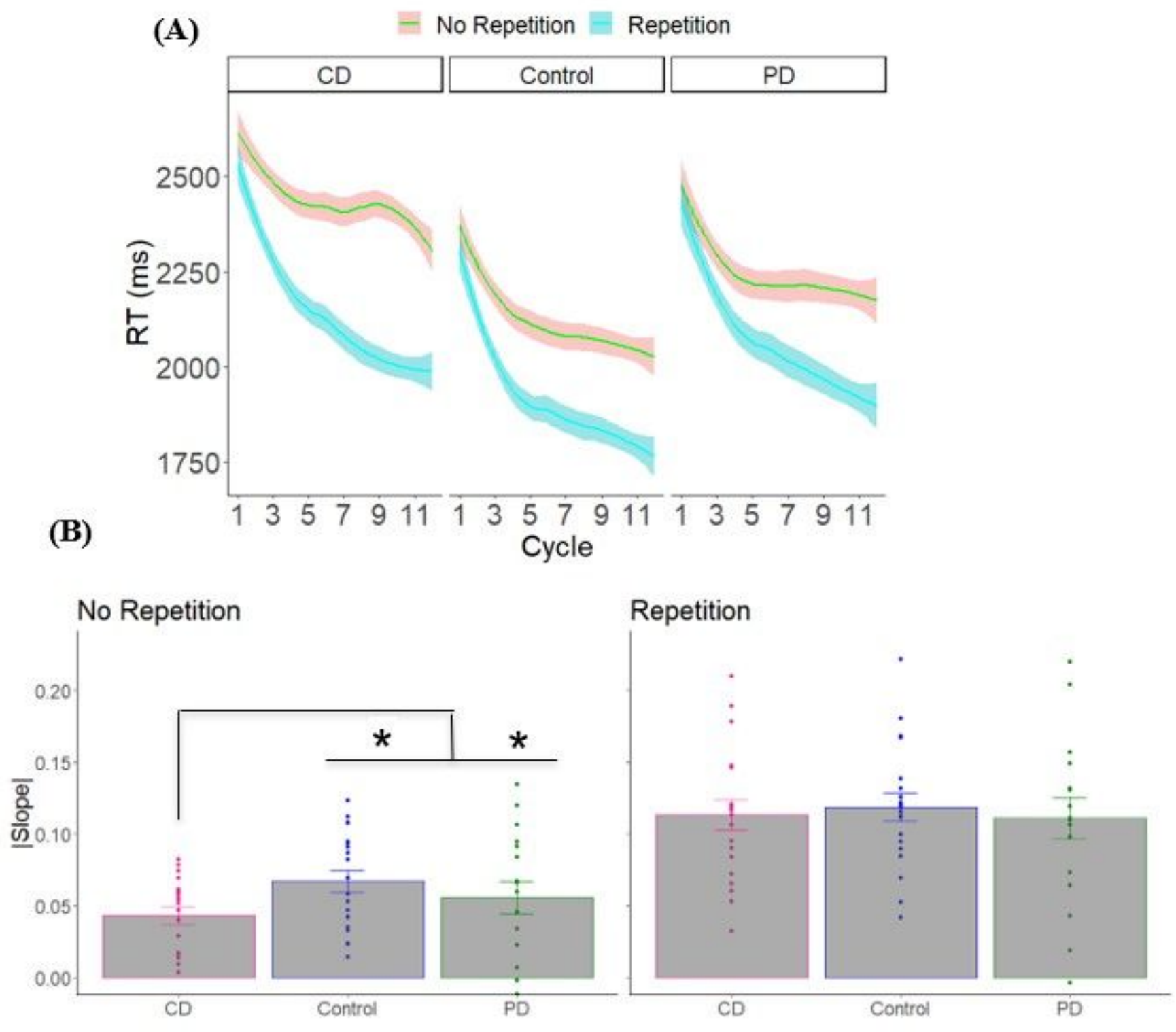

\section{Figure 2}

(A) RT as a function of learning cycle for the Repetition and No Repetition conditions. Error bars $=95 \%$ confidence interval. (B) Rate of improvement across cycles for each group for the No Repetition (left) and Repetition (right) conditions. Dots indicate performance of each individual participant. Error bars = SEM; ${ }^{*} \mathrm{p}<.001$. 